Results We received 53 responses from parents. 31(58.49\%) have neurodevelopmental disorder (autism spectrum disorder/ attention deficit hyperactivity disorder), 14(26.41\%) have learning disabilities, 5(9.43\%) have neuro disabilities (cerebral palsy/global developmental delay, and $3(5.66 \%)$ have other conditions such as chromosomal deletions.

27(50.94\%) parents felt that a virtual appointment with a paediatrician and nurses were very good and another 16 $(30.18 \%)$ felt it was good. $2(3.77 \%)$ of them felt it was neither good nor poor. None of them felt it was poor or very poor service but $8(15.09 \%)$ either did not answer this question or said I don't know. We also requested feedback from them about their experience and their comments are summarized in table 1.

Conclusions Overall, parents felt very much reassured that we are running a service during lockdown. However one parent felt that her child was too complex to be seen in virtual clinic. Our observations support the idea of multidisciplinary virtual clinics for special needs children but we agree that it cannot replace face to face consultations.

\section{PROFILES OF MALTREATED CHILDREN AGED 0-3 YEARS OLD AND THEIR FAMILIES: THE SINGAPORE EXPERIENCE}

Rui Kwan, Sharon Abraham, Wan Har Chong, Jean Yin Oh, Yeleswarapu Sita Padmini. Singapore

\subsection{6/bmjpo-2021-RCPCH.168}

Background Early adverse childhood experiences (EACES) such as child maltreatment between the ages of 0 to 3, has significant impact on a child's cognitive development and behaviour. Negative experiences and even the absence of positive experiences e.g. in extreme deprivation, can cause disruptions to brain architecture, with long-lasting effects. EACES also increase the risk of physical health, mental health and relational difficulties in adulthood with poor academic and employment outcomes. Understanding the risk factors associated with health and developmental conditions in maltreated children provide more effective interventions to improve their outcomes.

Objectives The purpose of the study was two-fold. One, to explore the demographics, social, developmental and physical health conditions of young children admitted to a tertiary children's hospital for suspected child maltreatment Two, to examine the occurrence of child maltreatment with developmental delays and missed vaccinations.

Methods 99 children aged 0 to 3 , admitted between the period of January 2018 and June 2019 were included in this quasi-observational study. Medical data was electronically extracted on variables surrounding their health, development, and demographics.

Results Children in this study had a mean age of 1.3 years; and 59\% were males. The most common reported abuse type was physical (74\%), followed by neglect (14\%) and sexual abuse (10\%) $2 \%$ had more than one of three abuse types. $35 \%$ of children had pre-existing developmental conditions and significant medical conditions e.g. asthma or atopy. During the hospitalisation, 58\% of children were assessed to have child maltreatment-related injuries, missed vaccinations and developmental conditions requiring health care followup. Specifically, 19\% $(n=19)$ of children had existing or present developmental delays, where six were detected through routine developmental screening during hospitalization. Children with developmental delays were more likely to have missed vaccinations than those without delays [Odds ratio (OR) 3.6, 95\% confidence interval (CI) 1.04-12.62]. The correlation with developmental delays was independent of maternal education or household income. Children staying in rented housing were more likely to have missed vaccinations than those staying in purchased housing [OR 9.5, 95\% CI 1.09-82.72]. When it came to parental factors, the majority of children's parents had only primary school education; and had a mean household income of $\$ \$ 823$ - well below the national average. $20 \%$ stayed in purchased housing, $27 \%$ in rented housing, and 5\% were lodging with relatives or friends. Majority of children (72\%) were referred to statutory child protection agencies as part of safeguarding procedures, indicating significant concerns over the child's injury and social circumstances.

Conclusions A sizeable proportion of maltreated children were identified with developmental delays - twice that seen in the general population. A significant proportion also presented with health concerns that utilize health care resources and follow-ups. Those with social deprivation were more likely to have neglected basic health needs e.g. vaccination. Thus, managing child maltreatment should include enhanced developmental screening using standardized tools to improve early detection and robust health surveillance, ensuring the timely identification of needs and access to appropriate services for better health equity.

\section{RENAL VASCULAR LESIONS IN CHILDHOOD-ONSET LUPUS NEPHRITIS: A TERTIARY CENTRE EXPERIENCE}

Eugene Yu-hin Chan, Alison Lap-tak Ma, Yuen-fun Mak, Wai-ming Lai. Hong Kong

\subsection{6/bmjpo-2021-RCPCH.169}

Background The clinical significance of renal vascular lesions remains unknown in childhood-onset lupus nephritis (LN) and is not currently factored into WHO and ISN/RPS classification.

Objectives The aim of this study is to determine the incidence and outcomes of childhood-onset LN in the presence of these lesions.

Methods We conducted a retrospective review at the tertiary paediatric nephrology centre in Hong Kong of children with LN who presented before 18 years with active follow-ups for 12 or more months by July 2020. Estimated glomerular filtration rate (eGFR) was calculated by modified Schwartz and CKD-EPI formula as appropriate.

Results Among 90 children with childhood-onset systemic lupus erythematosus, 61 patients $(68 \%$; mean age of 13.2 \pm 3.4 years; $85 \%$ girls) diagnosed to have biopsy-proven LN. The predominant presentation was nephritic-nephrotic syndrome $(n=30,50 \%)$, with 4 patients required acute dialysis. One (4\%), 17 (28\%), 29 (48\%), 9 (15\%), 4 (7\%) and $1(2 \%)$ patients had class II (lupus podocytopathy), III, IV, III/IV + V, $\mathrm{V}$ and VI LN, respectively.

Of the 61 patients with $\mathrm{LN}$, renal vascular lesions were observed in 9 children $(15 \%)$ at a mean age of $14.1 \pm 4.3$ years, including lupus vasculopathy $(\mathrm{LV} ; \mathrm{n}=4,7 \%)$, thrombotic microangiopathy (TMA; $n=4,7 \%)$ and true vasculitis $(n=1$, $2 \%$ ). Eight patients had co-existing proliferative LN (Class III 
$[\mathrm{n}=3]$; IV $[\mathrm{n}=3]$; mixed III/IV and V $[\mathrm{n}=2]$ ) and one patient had pure membranous LN. All children except two developed nephritic-nephrotic syndrome and AKI, and acute dialysis were initiated in 2 patients with TMA. At presentation, patients with renal vascular lesions had a significantly lower median eGFR (21.0, IQR 14.0-51.0 versus 88, IQR 48.5-107.5 ml/ $1.73 \mathrm{~m}^{2} / \mathrm{min} ; \mathrm{p}=0.011$ ) but similar degree of proteinuria $(3.1$, IQR 2.4-8.04 versus 2.5, IQR $1.3-5.1 \mathrm{mg} / \mathrm{mg}$ ) than those without such lesions.

Treatments were heterogeneous due to variable disease severity. In addition to pulse methylprednisolone, 6 and 3 patients received induction therapies with intravenous cyclophosphamide and mycophenolate mofetil, respectively. Three patients with severe AKI (LV $n=2$; TMA $n=1$; mean GFR $18.6 \mathrm{ml} / 1.73 \mathrm{~m}^{2} / \mathrm{min}$ ) responded to rituximab as add-on rescue therapies and eventually did not require acute dialysis. Therapeutic plasma exchange was performed in three patients with TMA as adjunctive therapies. Following induction, 6 patients attained complete remission and had normal GFR and no proteinuria at last follow-up. Three patients failed to respond and developed chronic kidney disease (CKD). One LV patient had poor adherence and repeated relapses leading to end stage kidney disease (ESKD) and received a kidney transplant. One patient with TMA and another with LV developed CKD II (GFR $74 \mathrm{ml} / 1.73 \mathrm{~m}^{2}$ / min) and CKD IV $\left(28 \mathrm{ml} / 1.73 \mathrm{~m}^{2} / \mathrm{min}\right)$, respectively. At the end of the study, more children with $\mathrm{LN}$ and renal vascular lesions developed either CKD or ESKD (3/6 [33.3\%] versus 4/48 [7.7\%], $\mathrm{p}=0.06$ ), although it did not reach statistical significance.

Conclusions Childhood-onset LN with renal vascular lesions had more severe presentation and may be associated with worse kidney outcomes. Identifying these lesions may have prognostic value and guide clinical management. Further largescale studies are required to define its role in the future LN classification.

\section{WEANING OFF INCUBATOR HUMIDIFICATION IN THE NEONATAL INTENSIVE CARE UNIT (NICU)- AN EVIDENCE UTILIZATION PROJECT}

Xia Wang, Soke Yee Lee, Tomomi Ogura, Sarasvati Rajoo. Singapore

\subsection{6/bmjpo-2021-RCPCH.170}

Background Infants born less than 32 weeks of gestation age are nursed in closed humidified incubator to minimise transepidermal water loss, prevent electrolytes imbalances and improve thermoregulation. The practice in the unit is these infants are cared for in closed incubators with $85 \%$ humidity for first 7 days of life, then from the second week onwards, gradually reducing the incubator humidity to $70 \%$, and from the fourth week, further decrease the humidity to $65 \%$. These infants are nursed in incubator with $65 \%$ humidity till they reached body weight of $1.7 \mathrm{~kg}$. The estimated length of stay in the humidified incubator for these infants is $1.5-3$ months. However, high or prolonged humidified environment is associate with Candida albicans, which has a reported mortality rate of $21-32 \%$ in very low birth weight infants. In 2015, two infants nursing in humidified incubator were detected having skin Candida albicans infection.
Objectives To minimise skin candida infection in infants requiring closed humidified incubator based on best available evidence.

Methods The length of stay in the humidified incubator and humidity setting were reviewed. In the new guideline, infant less than 32 weeks of gestation age is nursed in close incubator with humidity of $85 \%$ for the first 5 days of life. From the 6 th day onwards, to decrease incubator humidity by $5 \%$ daily, till it reaches the targeted discontinue setting of $60 \%$, which is 11 days of life. Five briefing sessions were conducted to all staff. Table of incubator humidity setting schedule are pasted in all incubators for easy reference. Staff compliance to new guideline was monitored daily. Project evaluation was by audits in April 2015 to June 2016.

Results The post-implementation and follow-up audit showed a $100 \%$ staff compliance. No candida infection was detected. The project has reduced the length of infant requiring humidified incubator to 10 days, which saved about SGD\$193.20 per infant per hospitalisation.

There were 244 infants (Year 2017: 52 infants, Year 2018: 68 infants, Year 2019: 59 infants, Year 2020: 65 infants) in total who were born less than 32 weeks of gestation age since project implementation to end of 2020. Further data showed there is no candida infection detected among these infants.

Conclusions The project provides clear humidity percentage guidelines for nurses to follow, and successfully achieved zero skin Candida infection on infants who are being nursed in closed incubator. The project provides clear humidity setting guidelines for nurses, and successfully achieved zero skin candida infection for infants who require close incubator.

\section{KNOTTED PERIPHERALLY INSERTED CENTRAL CATHETER IN A NEONATE}

Paula Tang, Yvonne Leung, Nicholas Chao, Michael Leung, Zita Hung, Kevin Fung. Hong Kong

\subsection{6/bmjpo-2021-RCPCH.171}

Background With the advance in technology and medical expertise, it has been increasingly common for clinicians to establish a secured vascular access in the hospitalized neonates. As the veins in preterm neonates are notoriously difficult to catheterize, clinicians are often left with limited options when a secured vascular access is clinically indicated for medications, fluids or intravenous nutrition.

Objectives Peripherally inserted central catheter (PICC) has the advantage of being easily available in most SCBU and NICU, does not require the input of anaesthetist or radiologist and they are often quite durable, lasting up to 3 to 4 weeks in a neonate with meticulous flushing and nursing care. While blood stream related infection, clot formation, malposition, occlusion and extravasation are commonly quoted PICC related complications in the literature, we report a case of tight knot formation of PICC during insertion in a neonate, subsequently requiring venotomy for retrieval.

Methods We report a case of a pre-term neonate with difficult PICC insertion and retrieval. Tight complex knotting of the PICC was confirmed by XRay, and the catheter was 